\title{
Comunicar riesgos en la sociedad de la incertidumbre
}

\section{Carlos Lozano Ascencio}

Doutor; Universidad Rey Juan Carlos, Madrid, Espanha

carlos.lozano@urjc.es

\section{Márcia Franz Amaral}

Doutora; Universidade Federal de Santa Maria, Santa Maria, RS, Brasil marciafranz.amaral@gmail.com

\section{Resumen}

Este texto ofrece una aproximación teórica para fundamentar un modelo de Comunicación Social de riesgos e incertidumbres que, por una parte, estructure las distintas etapas necesarias para delimitar y definir "el saber de la comunicación de riesgos e incertidumbres" $y$, por otra, contribuya al desarrollo de los planteamientos del Marco de Sendai para la Reducción del Riesgo de Desastres. A cada época le corresponden diversas formas de percibir y prever la inestabilidad social para denominarla y construirla significativamente como un espacio del entorno circundante que demanda de los sujetos la máxima atención. Actualmente, es habitual que se hable de los riesgos para caracterizar a nuestras sociedades, pero en esa clase de generalizaciones se suele aludir a situaciones de inestabilidad que, en el fondo, son mucho más complejas de lo que parecen y que no siempre se han delimitado ni se han podido controlar con la sola previsión. Los conocimientos y las tecnologías no han reducido los niveles de fragilidad social y, más aún, las apreciaciones que hacen los individuos a propósito de las situaciones inestables suelen incrementar históricamente los niveles de vulnerabilidad real, sobre todo porque se dejan influenciar por el punto de vista de los principales mediadores sociales.

\section{Palavras-chave}

Comunicación. Riesgos. Sociedad de la incertidumbre. Marco de Sendai. 


\section{Introducción}

Este artículo tiene dos partes bien diferenciadas: el punto de partida lo colocamos en lo que hace falta percibir y conocer antes de alcanzar la habilidad de comunicar; la idea es que antes de comunicar se aprende a percibir información de los entornos circundantes y que el sentimiento básico e instintivo del miedo es lo que impulsa e intensifica la evolución de la humanidad. La primera etapa culmina con las capacidades cognitivas que tienen los sujetos observadores frente a los peligros detectados en el aquí y en el ahora. La segunda parte desarrolla las prácticas habituales de mediación social para percibir, conocer y comunicar la indeterminación, es decir, las capacidades para detectar las mediaciones (individuales, sociales y mediáticas) de las situaciones de inestabilidad y fijarlas mediante discursos hegemónicos.

Al comunicar riesgos e incertidumbres se configura, en primera instancia, un "entorno de contraindicaciones", es decir, una reconstrucción narrativa que elaboran los sujetos primero, y los medios de comunicación después, al saber percibir, interpretar y poner en común los principales asuntos que suceden en el entorno y afectan directamente a la estabilidad de su dominio social de supervivencia. Mencionamos en este contexto el Marco de Sendai y mostramos cómo la comunicación aparece de manera difusa y general. Así, traemos algunos modelos de comunicación de riesgos para detallar algunas dimensiones de la comunicación del riesgo.

\section{Resultados: percibir y conocer antes de comunicar}

Primero hay que saber percibir la información del entorno circundante para poder comunicar sobre cualquier tema, dicha percepción de datos procedentes del exterior de los umbrales sensitivos de los sujetos favorece el desarrollo de determinadas habilidades cognitivas para saber procesar informaciónes. Dicho procesamiento de datos es la condición suficiente y necesaria para que los sujetos estén en condiciones de poder elaborar información nueva. Por consiguiente, sólo después de haber aprendido a desenvolverse como receptores de información los sujetos pueden convertirse en emisores de la comunicación (PIÑUEL RAIGADA; LOZANO ASCENCIO, 2006).

Los primeros grupos humanos, sin lenguaje ni conocimientos, fueron capaces, no obstante, de saber percibir de los entornos circundantes lo que más afectaba a su propia 
estabilidad (supervivencia) motivados por sus destrezas sensitivas y, sobre todo, por su innata capacidad para sentir y reconducir su miedo. Dicha perplejidad anímica los guiaba a recelar si sucedía algo diferente. El miedo ayudó a aquellos hombres a sobrevivir a los avatares y a evolucionar como especie.

\subsection{La evolución de una humanidad "miedosa"}

El miedo siempre ha estado presente a lo largo de la historia de la humanidad, desde las épocas más primitivas en las que los seres humanos vivían permanente atemorizados por cualquier tipo de avatar percibido en sus propios entornos circundantes, hasta nuestros días, en los que, curiosamente, también vivimos perplejos de manera continuada por toda clase de acontecimientos violentos y destructivos que propician nuevas situaciones de inestabilidad social.

No hay que olvidar que el miedo es esa clase de sentimiento desagradable y de desconfianza que compartimos con el reino animal; no obstante, el miedo en los animales, como apunta Delumeau (2002, p. 21) “[...] es único, idéntico a sí mismo, inmutable: el de ser devorado. Mientras que el miedo humano, hijo de nuestra imaginación, no es uno sino múltiple, no es fijo sino perpetuamente cambiante.". El miedo individual y el temor colectivo son dos afecciones consustanciales (históricas y antropológicas) tanto para la condición humana como para las situaciones de incertidumbre. De hecho, han sido (y son) dos testigos inseparables de la biografía de la cultura humana. El miedo no sólo presupone la alerta de los sentidos sino además la implicación y reconocimiento del sujeto por situarse en el interior de un estado proclive a la afectación. Cuando se tiene miedo no siempre hace falta saber a qué se teme, pero saber a qué se teme tampoco atenúa el miedo. Sólo en el caso de poder pronosticar una solución (real o inventada) a la situación de incertidumbre que se experimenta, estaríamos en el camino de poder apaciguar el miedo de los sujetos implicados, pero es probable que ni en ese caso se consiguiera. Por lo tanto, el miedo es una entidad tan subjetiva como irreflexiva.

¿Por qué no hemos sido capaces de erradicar por completo los miedos? La cultura humana se puede entender como una permanente e inacabada lucha declarada por los sujetos contra sus propios miedos; combate en el que, poco a poco, hemos ido ganando batallas a nuestros propios temores en tanto que hemos conseguido la expansión física y cultural de "dominios". En definitiva, dicha expansión física y cultural va construyendo 
escudos o caparazones que les sirven a los hombres para dominar e ir sometiendo al entorno a sus propios intereses de supervivencia.

Si en tiempos remotos los principales miedos de los hombres los acaparaban las fuerzas incontroladas e incomprensibles de la Naturaleza (bosques inexplorados, selvas impenetrables, mares inalcanzables) hoy en día, aunque no de manera exclusiva, todavía siguen estando vigentes esos miedos a los entornos circundantes a pesar del alto nivel de desarrollo que han alcanzado la ciencia y la tecnología. Al constatar que tanto los hombres primitivos como los contemporáneos comparten los miedos hacia los entornos, de formas muy diferentes, claro está (a pesar de que los primeros no tienen conocimientos y recursos para percibirlos y no obstante que los segundos tienen muchísima más información, conocimiento y recursos tecnológicos en el momento de percibirlos), es pertinente exponer la noción de dominio de supervivencia como una constante en la evolución de la humanidad movida por el miedo. Hay que subrayar en el hecho de que el miedo es una condición necesaria para que los sujetos detecten futuras amenazas y peligros.

\subsection{Los dominios de supervivencia o la previsión de amenazas}

Los "dominios" son las capacidades y/o habilidades que desarrollan y utilizan los seres humanos para conseguir la supervivencia frente a los avatares de los entornos habitados. Se trata, en primera instancia, de habilidades biológicas heredadas de la propia especie, también de destrezas cognitivas que se van adquiriendo con base en la experiencia para saber reconocer y anticiparse a los trastornos de los entornos $\mathrm{y}$, por último, son capacidades culturales aprendidas en el seno de las relaciones sociales. En otras palabras, los territorios y trayectos de los dominios le permiten a los sujetos existir y sobrevivir biológica y socialmente; fuera de dichos dominios no existen ni los sujetos ni se perciben los entornos circundantes; biológicamente el ser humano no puede desarrollar esquemas de actuación, interacción y comunicación sin el acoplamiento estructural que le viene impuesto por regulaciones que él no se inventa, sino que le preceden y tiene que aprender a partir de la escasa dotación genética con la que nace; el acoplamiento a la estructura social de los dominios perdura y evoluciona en la medida en que el resto de sujetos individuales participan decididamente en el mantenimiento y transformación del dominio frente a los acontecimientos imprevistos. 
Es verdad que la sola existencia vital de los seres humanos implica su supervivencia, pero este último término recrea, con más detalles, la idea de haber experimentado incidentes peligrosos que pudieron atentar contra su vida y contra su continuidad en los entornos habitados y regulados por los dominios. Si un ser humano "existe" no necesariamente indica que sea capaz de sobrevivir a las eventualidades; sin embargo, si un ser humano "sobrevive", por lo menos se alude a ese esfuerzo mínimo necesario que necesitan llevar a cabo los sujetos para alcanzar el objetivo de seguir estando y desarrollándose en el mundo que les ha tocado (no sólo vivir, sino) sobrevivir. De aquí que podamos hablar de la "supervivencia" para subrayar aquellas facetas básicas que han facilitado a los sujetos, desde los más primitivos a los del mundo contemporáneo, a relacionarse con los entornos circundantes en función de cambios, incidencias, emergencias e incertidumbres y no en función de estabilidades, equilibrios y certezas.

Los dominios de supervivencia no sólo se han construido a lo largo del tiempo de acuerdo con el capital cognitivo disponible en cada época histórica, sino que también han mantenido latentes (o incluso intactas) algunas habilidades básicas (más bien de carácter instintivo e irracional) que les han permitido a las víctimas de catástrofes desenvolverse intuitivamente tan sólo para buscar la supervivencia a cualquier precio. En definitiva, dichos dominios facilitan la resiliencia o el incipiente control de los avatares del entorno circundante, y dicho control se manifiesta con la identificación de los "entornos concernientes", del aquí y ahora de aquellas partes o fragmentos específicos del acontecer (peligros) con los que los sujetos se sienten mucho más implicados porque les afectan directamente.

\subsection{La percepción de los peligros que más atentan contra la supervivencia: la configuración de los entornos concernientes}

El entorno natural es diverso, complejo y caótico hasta que los sujetos que lo habitan reducen los niveles de incertidumbre. La reducción de incertidumbre comienza cuando los sujetos son capaces de percibir los estímulos procedentes del entorno natural (estímulos de producción autógena). Para que los sujetos perciban dichos estímulos, éstos tienen que estar, necesariamente, al alcance de sus campos sensitivos; los estímulos de producción autógena que estén fuera de los campos sensitivos de los seres vivos no pueden ser percibidos sencillamente por razones físicas; ahora bien, no todos los estímulos de 
producción autógena son percibidos aun estando dentro de los límites del campo sensitivo, ¿por qué sucede esto? Porque no todas las estimulaciones autógenas comprometen la estabilidad o supervivencia de los sujetos. Los estímulos autógenos que comprometen la estabilidad o supervivencia de los sujetos no sólo se perciben, sino que, sobre todo, es lo primero que se identifica no sólo porque es lo que más les llama la atención, sino, sobre todo, porque atentan contra su vida, les provocan miedo y angustia, y desatan reacciones para ajustarse a las eventualidades del entorno.

Las estimulaciones autógenas percibidas (atendidas, registradas, adaptadas) por los sujetos acarrean, necesariamente, ajustes de conducta, es decir, lo que hacen una vez lo vuelven a hacer en situaciones similares, y sólo hasta que dichos ajustes de conducta se repiten reiteradamente, los sujetos comienzan a crear esquemas de conductas que, con el tiempo, son capaces de utilizar como reacciones resilientes (ya previstas) a las estimulaciones autógenas más conocidas. Así pues, las habilidades de percepción de los entornos, a partir de aquellas estimulaciones autógenas tan comprometedoras como progresivamente conocidas por los seres vivos, promueven la creación de esquemas o pautas de conductas para reaccionar con éxito e incluso reaccionar de manera anticipada; este éxito en la creación de dominios de supervivencia asegura dos cosas muy importantes: a) mayor estabilidad y permanencia de los sujetos en su hábitat (supervivencia); y b) más habilidad por parte de los sujetos para manejar información (comunicación).

Manejar información es la capacidad para ordenar las estimulaciones autógenas como datos susceptibles de ser utilizados para tomar decisiones, conocer mejor el entorno circundante en función de lo que más les concierne y les interesa para poder identificar (percibir), interpretar (conocer) y comunicar (compartir) los principales peligros a los que están expuestos en el aquí y ahora de su dominio de supervivencia.

\subsection{El sujeto observador frente al peligro observado en el "aquí y ahora"}

Con el tiempo, la sofisticación de los modos de sobrevivir trajo consigo una depuración en las formas de vida y dentro de dichas depuraciones podemos destacar la percepción que hicieron los seres humanos de "sí mismos", reconociéndose como un "sujeto observador y conocedor" diferenciado del colectivo, un primer paso en el origen individual del "yo psicológico". 
"Percibirse y conocerse a sí mismos "significa que los humanos primitivos al olerse, tocarse, oírse, probarse, verse, terminaron por sentirse a sí mismos (inicio de la conciencia individual) y sentir el mundo exterior desde dentro de sus cuerpos (descubrimiento cognitivo del "yo psicológico"). Lo anterior supone que desde el momento en que lo seres humanos pudieron tener conciencia de sí mismos, convirtiéndose en sujetos, en "seres conocedores", fueron aprendiendo a ver el mundo no sólo con los ojos, sino, sobre todo, con puntos de vista, es decir con "miradas subjetivas" para distinguir, ajustarse mediante esquemas consolidados de conducta y modificar el entorno natural en función de sus propios intereses de supervivencia.

El origen del conocimiento, de saber hacer lo necesario para sobrevivir, fue una consecuencia de las tensas relaciones entre sujetos y entornos circundantes. En la medida en que los hombres fueron aprendiendo a hacer cosas para sobrevivir frente a las inclemencias medioambientales, y también en la medida en que lo consiguieron, fueron capaces de introducir un orden o una estructura de clasificación para comprender (mediante explicaciones ficticias primero y mucho más adelante con respuestas científicas) lo que sucedía a su alrededor, y después para actuar sobre el entorno manipulando y modificando los materiales que tenían a su alcance. Cuando el ser humano "conoce" el entorno natural, cuando es capaz de diferenciarse frente al entorno. Simultáneamente al nacimiento (epistemológico) del sujeto surgen los "objetos", esto es, todo aquello que conoce el sujeto, cualquier material, fenómeno, situación, eventualidad, entidad animada, etc., a los que el sujeto dirige su mirada y/o transforma con sus manos.

Si los sujetos perciben los peligros con los que se sienten mucho más implicados, sentirán básicamente miedo y ubicarán esos peligros en un escenario espacio-temporal cercano. Ahora bien, no sólo el miedo instintivo es el único impulsor de la percepción cada vez más selectiva de la incertidumbre; se suman la experiencia y el saber hacer lo necesario para mantener la estabilidad mediante el control (conocimiento y previsión) de los principales peligros. Es decir, el primer ejercicio de conocimiento generalista que llevan a cabo los sujetos cognoscentes es precisamente buscar una respuesta satisfactoria para creer que conocen las causas de los principales peligros que atentan contra su propio dominio social de supervivencia.

Las mejores explicaciones para conocer el origen de los peligros más acuciantes no estaban dentro de los márgenes de los dominios de supervivencia, sino fuera, muy lejos, a tanta distancia como los sujetos fueran capaces de trasladar sus meditaciones y 
especulaciones sobre esos asuntos. Querían anticiparse a un posible daño de futura ocurrencia que seguramente surgiría por las fuerzas incontroladas de la naturaleza, sin que los sujetos tuvieran algo que ver con ello.

En la medida en que la humanidad comenzó a incorporar el conocimiento experimental disponible (contrastable y objetivable) para percibir e interpretar los peligros, se inició un largo camino para saber anticiparse a la incertidumbre; por lo tanto, si a cada época histórica le correspondía un nivel determinado de desarrollo científico y tecnológico, también le correspondía una manera determinada de percibir, configurar comunicativamente las contraindicaciones del entorno y prever la inestabilidad. Puede ser cierto que hoy en día identificamos muchos más peligros que antes, pero el avance del conocimiento científico nos obliga a saber mucho más de dichas eventualidades y en tal caso poder identificarlas como vulnerabilidades o riesgos, esto es, como amenazas mucho más sofisticadas y, por supuesto, mucho más previsibles.

Figura 1 - Evolución histórica de la percepción de las situaciones de inestabilidad: de la implicación individual, la cercanía y el desconocimiento al reconocimiento social, la lejanía y el conocimiento.

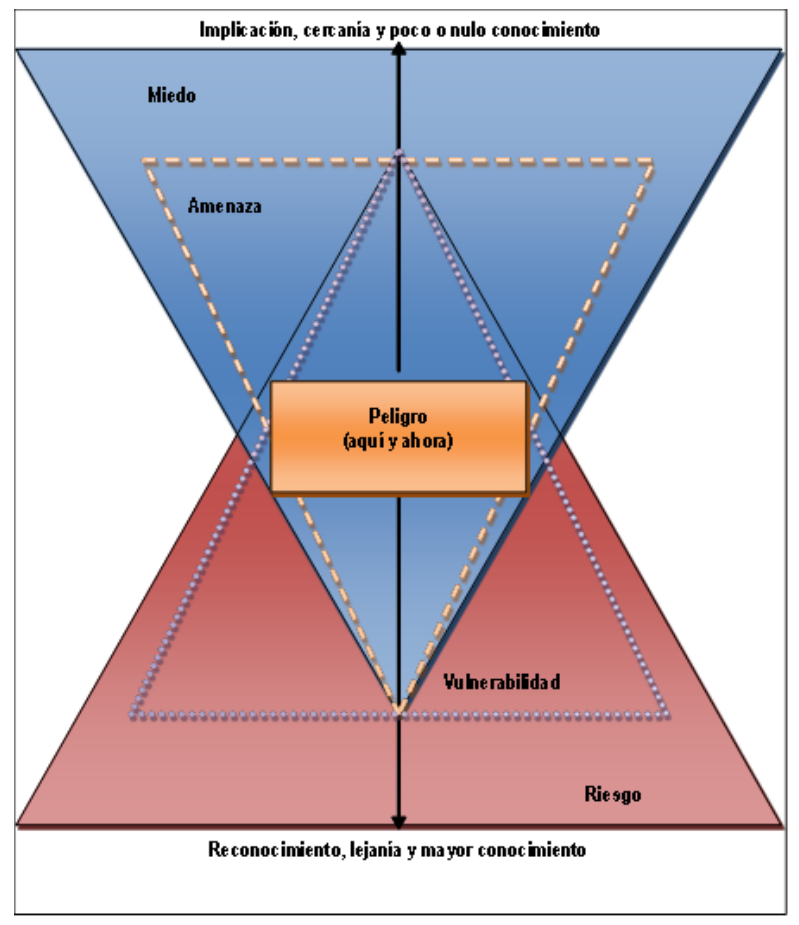

Fuente: Elaboración propia.

La percepción de los peligros, según aparece en la Figura 1, es la acción más recurrente que puede estar presente en todas las épocas históricas, en tanto que obliga a los 
sujetos a percibir las situaciones de inestabilidad en el aquí y ahora, manteniendo un equilibrio entre lo que se ignora y se sabe, entre lo que atañe al sujeto y a su comunidad, entre lo sucedido ayer y lo que pueda suceder mañana y entre lo más próximo y lo más lejano. La diferencia estriba en que, en función de los conocimientos socialmente disponibles en cada época y lugar y de los niveles de comunicación consolidados socialmente, los sujetos pueden interpretar las inestabilidades proyectando sus miedos o sus certezas para entender y anticiparse a los peligros vigentes de su época. En la Figura 1 se puede apreciar que "Percibir peligros aquí y ahora" son las distintas formas en las que los sujetos detectan las situaciones de inestabilidad en su momento vigente, y dichas percepciones no están exentas de mezclar, en distintas gradaciones, la implicación individual y el reconocimiento social, la cercanía y la lejanía, el desconocimiento y el conocimiento, la imprevisión y la previsión, las formas básicas y las formas más sofisticadas de comunicarse. También, en la medida en que los sujetos se sientan más implicados y cercanos con la urgencia de la inestabilidad y que además se orienten básicamente por sus propias motivaciones instintivas, detectarán la inestabilidad como un producto de sus propios miedos y, lo contrario, en la medida en que los sujetos tengan la capacidad de interpretar la urgencia de la inestabilidad con mayores conocimientos tendrán más opciones de orientar su decisiones y acciones para atenuar e incluso para saber prever que dicha situación no desemboque en consecuencias mayores.

\section{Los entornos de contraindicaciones: percepción, conocimiento y}

\section{comunicación de la inestabilidad}

Los entornos de contraindicaciones son reconstrucciones narrativas que elaboran los sujetos primero, y los medios de comunicación después, al saber percibir, interpretar y poner en común los principales asuntos que suceden en el entorno que afectan directamente a la estabilidad de su dominio social de supervivencia. Se podría decir que son núcleos de sentido que se extraen de los entornos concernientes como señales de alarma, avisos disuasorios, advertencias, notificaciones, indicaciones, alertas sobre peligros inminentes o riesgos más probables.

Los entornos de contraindicaciones se distinguen y se delimitan, del resto de los entornos, mediante marcas rotuladas con colores llamativos, algo parecido a lo que hace la 
policía cuando acordona una zona para que nadie traspase dichas marcas. Las cintas fluorescentes advierten "no pasar" pero en dicho apercibimiento no hay más información que revele las causas del aislamiento; no obstante, se establece una convención, respetada por quienes están próximos o se sienten implicados con lo que sucede. La elaboración narrativa de una contraindicación de lo que sucede en el entorno está al alcance de cualquiera que sepa percibir y comunicar peligros, ahora bien, es obvio que la narración de esas contraindicaciones en un principio se hicieron con conocimientos generalistas, básicos, sin muchos fundamentos $y$, con el paso del tiempo, como es de esperar, se impusieron en los discursos sociales no sólo como aportaciones del conocimiento científico, sino, sobre todo, por la imposición discursiva de los medios de comunicación en estos asuntos.

\subsection{La percepción de las situaciones de inestabilidad}

Las situaciones de inestabilidad son aquellos particulares estados de la realidad social en los que los sujetos que se encuentran inmersos en ellos son incapaces de identificarlos y definirlos con claridad; en esas circunstancias lo que más destaca es la indeterminación y el sentimiento de inseguridad para quienes intentan darles algún sentido. Se establecen cuando los sujetos sienten la perplejidad de dicha circunstancia; no cobra sentido su "sin sentido" hasta que los sujetos implicados pueden contraponer o asociar lo que perciben con lo que ya conocen o creen conocer; $y$, finalmente, si los sujetos son capaces de controlar dichas situaciones no lo consiguen por saber "qué es lo que pasa", sino más bien por saber lo contrario: "qué no es lo que pasa".

Hay que tener en cuenta que tanto la incertidumbre como el riesgo son situaciones de inestabilidad, sin embargo, son muy diferentes entre sí. La incertidumbre se caracteriza por ser una situación en la que los sujetos involucrados sólo detectan la irresolución de lo que sucede o la resolución de lo que no sucede porque cuentan con poca información y escaso conocimiento para saber contrarrestar la desazón que experimentan. En tales circunstancias lo sujetos no tienen certezas de lo que puede pasar, y de llegar a tenerlas desconocen las probabilidades de su ocurrencia, no lo pueden calcular. En cambio, en una situación de riesgo, los sujetos involucrados cuentan con más información y conocimiento para saber salir de dicha situación, tienen más certezas de lo que puede pasar y, de no contar con certezas, tienen más conocimiento de las probabilidades de lo que puede ocurrir, es decir, pueden calcular el desenlace más probable. Si en una situación de riesgo los sujetos 
involucrados pueden prever la ocurrencia de un trastorno perjudicial se debe, exclusivamente, a la información y conocimientos acumulados con los que disponen en ese momento. Por consiguiente, la ubicación de las situaciones de riesgo es una delimitación estrictamente perceptiva de atribuciones especulativas que realizan los sujetos al intentar interpretar la inestabilidad o anormalidad de los entornos que más conciernen. Así, si el grado de importancia de los riesgos viene determinado directamente por los niveles de información y conocimiento de los sujetos observadores, se puede afirmar que sin éstos, sujetos informados y conocedores, no puede haber riesgos. El riesgo es la medida consensuada de la incertidumbre. Medir la incertidumbre es una manera de especificar, delimitar, conocer y reconocer con más detalles los indicadores de la fragilidad, es decir, la existencia y detección de un riesgo implica el conocimiento necesario para establecer una articulación entre las posibles causas de la vulneración, las situaciones vulnerables y las consecuencias que inevitablemente tendrán la condición de haber sido vulneradas con respecto a su estado inmediatamente anterior. Hablar de riesgos compromete a los interlocutores porque es posible deducir que algo saben a propósito de que algo peligroso (o catastrófico) puede suceder.

Entre la incertidumbre (inestabilidad imprevisible) y el riesgo (inestabilidad previsible) existen muchos matices que gradúan la percepción de las situaciones de inestabilidad; de hecho, los individuos y las sociedades utilizan diferentes clases de herramientas biológicas, técnicas y sociales que les permiten conocer, paso a paso, lo que ocurre en sus entornos para saber actuar sobre ellos, ya sea para modificarlos o, sencillamente, para sobrevivir a sus envites. Ahora bien, se podría decir que un mayor nivel de conocimientos disponibles se correspondería con mayores posibilidades (técnicas y culturales) de saber prever riesgos, que el avance de la reflexividad acarrearía necesariamente un control más efectivo frente a la vulneración de los entornos, no obstante, nuestras sociedades modernas, las llamadas "sociedades del riesgo" no sólo son más frágiles a padecer trastornos destructivos, ni se caracterizan sólo por generar cada vez un mayor número de inestabilidades previsibles, sino que el avance disponible de la ciencia y la tecnología no es suficiente para prever, evitar, ni administrar los riesgos tangibles, dado que siempre hay márgenes de imprevisión que vuelven a impregnar de incertidumbres la realidad estudiada. Daría pues, la sensación de que el proceso histórico que ha llevado a la Humanidad y al conocimiento científico a discernir entre las situaciones previsibles e imprevisibles de inestabilidad, vuelve al punto de partida y que hoy en día nos enfrentemos 
a nuevas situaciones de "riesgo incontrolable" como rasgo idiosincrásico de la "sociedad de la incertidumbre".

Si la previsión de las situaciones de inestabilidad depende más del nivel de información y conocimientos disponibles en determinados momentos y/o escenarios concretos, que de la visibilidad, proximidad o inminencia de aquello que tiene la facultad de modificar destructivamente la realidad, es obvio que si no se tiene información ni se sabe nada sobre la ocurrencia de una catástrofe segura nos encontramos ante un dilema: o la destrucción se anticipó inesperadamente, nadie sabía ni preveía que podría ocurrir, o lo que en realidad sucede, en tanto que no es previsto por nadie carece de interés, que es tanto como decir: "aquí no ha pasado nada".

\subsection{La certeza de la fragilidad social a los riesgos}

En la medida en que aumenta la confianza en las explicaciones disponibles sobre el entorno, así como en las actividades con las que los sujetos ponen en práctica sus conocimientos, tendríamos mejores opciones para poner barreras a la incertidumbre. Pero el avance en el conocimiento disponible no sólo está en condiciones de reducirla, sino también de incrementarla. Saber más acerca del entorno conlleva saber cuándo se está más expuesto al peligro y por lo tanto cuando existen potenciales amenazas frente a las eventualidades del entorno. Conocer más a fondo las amenazas determina la configuración más detallada de la vulnerabilidad o predisposición (física, económica, política o sociológica) que tiene una comunidad de sufrir daños en caso de que un fenómeno autógeno o antrópico pueda manifestarse. En este contexto, vale la pena hacer notar que la diferencia entre estar amenazado y ser vulnerable se puede explicar mediante una graduación de conocimientos sobre los avatares del entorno.

Al incrementar los niveles de información y conocimientos no sólo mejoran los formatos narrativos que reconstruyen y explican las quiebras del acontecer, sino que, sobre todo, se van imponiendo hegemónicamente en las prácticas sociales protocolizadas para saber ubicarse, prever y gestionar las quiebras del acontecer, esta supremacía discursiva incluso puede desembocar en relatos ejemplares o canónicos en los que los mayores niveles de información y conocimiento facilitan la existencia de esquemas sociales de previsión y afrontamiento. 
Habría que reconocer que si dicho avance de la reflexividad social estuviera aparejado al manejo de los discursos utilizados por los medios de comunicación sabríamos que las últimas propuestas científicas, en relación a la prevención de situaciones de inestabilidad, aparecerían con mucha más frecuencia en los relatos de la actualidad informativa; todos sabemos que no es así, que, como mucho, el mayoritario discurso genérico sobre el riesgo más bien impone "llaves interpretativas" para poder distinguir y entender un poco mejor el desorden.

Actualmente, el cambio experimentado en la percepción social de los peligros, frente a las quiebras del acontecer, obliga a los sujetos a esforzarse a mantener un constante equilibrio entre los peligros que más les atañen y les comprometen frente a los peligros que les parecen razonablemente importantes y relevantes, aunque con éstos últimos no necesariamente se sientan implicados. La diferencia es clara, mientras predomine la urgencia los sujetos saben con claridad cuál es el peligro que más les incumbe, en tanto que si predomina la complejidad los sujetos deciden u opinan sobre el peligro más relevante. Históricamente, los discursos genéricos disponibles (mediáticos o no) sobre las quiebras del acontecer se han impuesto de forma hegemónica a la reflexividad de los sujetos, en algunas ocasiones como producto de ideas doctrinarias dominantes, en otras como resultados de hábitos culturales que terminan extendiéndose y asentándose en el imaginario escatológico de las sociedades.

Los discursos hegemónicos, sobre las quiebras del acontecer, realizan previsiones para confirmar las vulnerabilidades de algún estado de la realidad social, apelando al conocimiento experto (científico) o no experto (genérico) con tal de centrar la atención en la existencia de futuras situaciones reales de riesgo; en este sentido, cuando los discursos hegemónicos desembocan de manera fehaciente y contrastada en una previsión inequívoca de riesgos inminentes, dichos discursos se convierten en relatos más bien ejemplarizantes (canónicos) que la sociedad en su conjunto se ve en la necesidad imperiosa de atender, aunque en realidad sean los sujetos mayor implicados y concernidos los que más atención prestan, para adoptar respuestas organizadas (protocolarias) y saber lo que se tiene que hacer para atenuar, afrontar, administrar o, en caso extremo, huir de esas situaciones de riesgo.

Es cierto que a mayor grado de reflexividad en los discursos sobre las quiebras del acontecer mayor es el nivel de cientificidad y ejemplarización protocolaria, sin embargo los medios de comunicación en sus discursos informativos genéricos (y generalistas) a 
propósito de estos asuntos no suelen utilizar unilateralmente esa clase de relatos tecnocientíficos, más bien abordan los acontecimientos apelando al conocimiento (experto y no experto) socialmente disponible con tal de despertar el interés de los espectadores y hegemonizar las reconstrucciones narrativas y escénicas de las quiebras del acontecer; en este sentido, los mencionados cambios en la percepción social de los peligros no sólo es un permanente conflicto de los sujetos entre la implicación y complejidad de lo que pasa, sino también los medios de comunicación, al abordar narrativamente las quiebras del acontecer, intentan mantener su particular equilibrio entre la apelación a los instintos más básicos de los sujetos y la apelación a su reflexividad cognitiva para detectar las fragilidades o riesgos más previsibles.

Gil Calvo (2009, p. 188) resume la relación entre riesgos y medios de comunicación afirmando que: “[...] si la inseguridad se incrementa no es sólo porque la realidad social genera mayores niveles de riesgo sino también porque los medios informativos transmiten mayores niveles de alarma social.”. ¿El discurso científico está tan se alejando que su utilización no facilita la inteligibilidad de las quiebras del acontecer o es que nos encontramos en una época donde también los conocimientos son frágiles?

Los medios de comunicación intervienen, sobre todo, en la imposición de discursos hegemónicos que construyen y actualizan continuamente las imágenes de la fragilidad social, y también hacen uso, aunque en menor medida, de discursos canónicos que contribuyen a establecer normas para afrontar los riesgos sociales más previsibles; hay que decir que cuantas más referencias al peligro se propone y cuantas más percepciones de amenazas se representan en sus relatos se generan reacciones básicas de miedo en aquellas personas más desvalidas ante la complejidad de los discursos. En suma, ni los discursos científicos ni los discursos mediáticos elaboran sus reconstrucciones narrativas sobre las quiebras del acontecer motivados sólo por la urgencia o sólo por la complejidad, ambos necesitan que sus discursos mezclen diferentes intervalos de implicación y reflexividad.

\subsection{Propuestas y retos de la comunicación del riesgo en un marco específico y sus dimensiones}

Ya hemos dicho que a cada época histórica encontramos maneras diferentes de percibir y manejar los riesgos. Dentro de estas perspectivas está la que protagoniza la Tercera Conferencia Mundial de la ONU dedicada a la Reducción del Riesgo de Desastres 
(2015-2030), realizada en 2015, en Sendai, Japón, y que ha sido adoptada por 187 países. El Marco de Sendai indica en que términos los países deben tratar los riesgos y señala algunas funciones de la comunicación en este proceso. Se trata, por tanto, de una respuesta organizada a la percepción del riesgo, pero se hace necesario detallar el papel de la comunicación.

El objetivo del documento de Sendai es sistematizar los aspectos para la reducción de riesgos de desastres de manera concisa, centrada y orientada en la acción. El documento busca intervenciones más específicas y multisectoriales en la lucha contra los factores que facilitan la aparición de desastres, como la pobreza, la desigualdad, la mala gestión del suelo, la variabilidad climática y la mala planificación urbanística. Considera que la reducción y la gestión del riesgo de desastres dependen tanto de los mecanismos de coordinación como de la necesidad de desarrollar ciudades resilientes. Sus objetivos son: entender los riesgos, fortalecer la gestión de los mismos, invertir en sus reducciones, en la resiliencia y en reforzar la prevención de desastres con respuestas efectivas.

Acerca de la comunicación, de manera amplia, el documento evidencia que:

Los medios de comunicación deben desempeñar un papel activo e inclusivo a nivel local, nacional, regional y mundial contribuyendo a la sensibilización y entendimiento públicos y difundiendo información exacta y no confidencial sobre los riesgos de desastre, las amenazas y los desastres, incluidos los desastres en pequeña escala, de una manera sencilla, transparente, fácil de entender y accesible, en estrecha cooperación con las autoridades nacionales, aprobar políticas de comunicación específicas para la reducción del riesgo de desastres, apoyar, como corresponda, los sistemas de alerta temprana y las medidas de protección y salvamento, y estimular una cultura de prevención y una fuerte participación de la comunidad en campañas de educación pública sostenidas y consultas públicas a todos los niveles de la sociedad, de conformidad con las prácticas nacionales (MARCO DE SENDAI..., 2015, p. 24).

Entre los principios rectores acerca de la comunicación están “[...] utilizar las innovaciones en materia de tecnología de la información y las comunicaciones para mejorar los instrumentos de medición y la recopilación, el análisis y la difusión de datos.” y “[...] reforzar la utilización de los medios de comunicación, incluidas las redes sociales, los medios tradicionales, los macrodatos y las redes de telefonía móvil, en apoyo de las medidas nacionales para una comunicación efectiva de los riesgos de desastres [...]." (MARCO DE SENDAI..., 2015, p. 15). El Marco presupone también:

[...] la evaluación periódica y la comunicación pública de los avances en los planes nacionales y locales y promover el escrutinio público y alentar los 
debates institucionales, en particular entre legisladores y otros funcionarios pertinentes, sobre los informes de los avances en los planes locales y nacionales para la reducción del riesgo [....] (MARCO DE SENDAI..., 2015, p. 17).

El documento trata todavía de la necesidad de desarrollar sistemas de alerta temprana y de predicción de amenazas múltiples:

[...] que sean multisectoriales y estén centrados en las personas, mecanismos de comunicación de emergencias y riesgos de desastres, tecnologías sociales y sistemas de telecomunicaciones para la supervisión de amenazas, e invertir en ellos; desarrollar esos sistemas mediante un proceso participativo; adaptarlos a las necesidades de los usuarios, teniendo en cuenta las particularidades sociales y culturales, en especial de género; promover el uso de equipo e instalaciones de alerta temprana sencillos y de bajo costo; y ampliar los canales de difusión de información de alerta temprana sobre desastres naturales. (MARCO DE SENDAI..., 2015, p. 21).

Se observa que la comunicación tiene presencia muy generalista y difusa en el texto del Marco y es necesario organizar y detallar progresivamente sus dimensiones y papeles. Según las aportaciones de Victor (2015, p. 10, grifo nosso), la comunicación en estas situaciones demanda "[...] efetiva interação e diálogo entre os interlocutores, impedindo o surgimento de fenômenos que ampliam socialmente os riscos, como rumores, pânico, desorientação e desconfiança.". Sistematiza cuatro modelos de la comunicación: intrainstitucional, interinstitucional, comunitario y mediático. Son modelos que deben ayudar a las tomas de decisiones más horizontales, reducir el miedo, orientar la información, atender a las demandas de los medios de comunicación y mejorar la comunicación entre las varias instituciones involucradas.

Observamos que estos modelos funcionan como niveles específicos de comunicación que poseen exigencias diversas. Ejemplificamos estos cuatros modelos con base en la realidad brasileña en que la protección y defensa están organizadas bajo un sistema llamado Sistema Nacional para la Protección y Defensa Civil, compuesto por un conjunto de organismos multisectoriales. El modelo intrainstitucional es cuando la Defensa Civil promueve acciones de comunicación de riesgo para el público interno. El modelo interinstitucional fomenta la participación de diversas instituciones directa o indirectamente actuantes en la gestión de riesgos con el fin de lograr una comunicación integral del riesgo. Los interlocutores que dialogan con la Defesa Civil están en los niveles Ejecutivo y Legislativo y también en instituciones técnicas, científicas, educacionales, religiosas, de salud y seguridad. La comunicación del riesgo se lleva a cabo también en el 
ámbito de las comunidades (en directo), cuando la Defensa Civil desarrolla acciones más primarias de comunicación sin la utilización de los medios, o sea, va directamente a la comunidad y las personas en situación de riesgo.

En el ámbito mediático, que nos interesa particularmente, la comunicación de riesgo ocurre cuando instituciones, como la Defensa Civil, necesita recurrir a los medios para diseminar informaciones o el proceso inverso también se produce, es decir, los medios de comunicación recurren a la Defensa Civil y recogen informaciones para aclarar un riesgo particular para la población. En este caso, para Victor (2015), la Defensa Civil se refiere al ámbito de aplicación, el poder de la ubicuidad, la velocidad y la credibilidad de los medios de comunicación para difundir información a un público más amplio, heterogéneo, con todo conectado por la misma preocupación.

Como se puede observar, hay varias dimensiones de la comunicación en este proceso. Incluso en el ámbito de los medios de comunicación las acciones están estrechamente vinculadas a una red de instituciones y actores que tienen el conocimiento sobre el acontecimiento. Es decir, los medios no pueden actuar aislados. Para establecer un vínculo entre las causas y consecuencias y mapear vulnerabilidades, los medios de comunicación necesitan superar el enfoque periodístico tradicional centrado en el día del desastre con la mera información fáctica y también invertir en los riesgos particulares y debates como el desarrollo de las ciudades más resilientes. Además, los medios deben estar en sintonía estratégica con las otras instancias (comunicación intrainstitucional, interinstitucional y para comunidades) de modo que la comunicación pueda centrarse en el antes y el después de los desastres y sobre todo al riesgo y no sólo en el caso estricto del desastre en particular. Esto requiere mucho más preparación del campo comunicacional por parte de los grandes medios.

\section{Conclusiones}

El riesgo es la detección más precisa de la incertidumbre que delimita, con más detalles, los indicadores de la vulnerabilidad. Aunque se trata de la posición más avanzada y compleja de los sujetos frente a la incertidumbre o el peligro (Figura 1), puede tener una implicación muy baja (no da miedo) porque se puede llegar a interpretar como algo que está francamente lejos en el tiempo y en el espacio.

Contemporáneamente, el ámbito mediático tiene papel decisivo en la percepción de los peligros. La actualidad informativa sobre los riesgos normales (previsibles) y los riesgos 
excepcionales (imprevisibles) se sustenta en las reconstrucciones escénicas de las quiebras del acontecer, se trata de discursos genéricos y muy amplios que describen, con meridiana claridad, lo sucedido; su estructura narrativa se caracteriza por ser un esporádico goteo de datos que, con el paso del tiempo, llega a convertirse en un gran torrente de información que desborda, por acumulación, la percepción y comprensión de lo que realmente ha ocurrido y está ocurriendo; de hecho, dicha estructura narrativa funciona exactamente al revés de lo que ocurre en la realidad, en donde el riesgo previsible o imprevisible se manifiesta a partir de un gran torrente de inestabilidad novedosa e ininteligible que, pasado el tiempo, se va estabilizando en una nueva situación de calma.

Los medios de comunicación no aportan información rigurosa con los discursos genéricos de las contraindicaciones de las quiebras del acontecer, más bien, facilitan el acceso de un gran número de gente que experimenta la inestabilidad de manera simultánea y, en consecuencia, contribuyen a cambiar la "percepción social de los peligros". Las nuevas tecnologías eliminan las barreras del tiempo y el espacio y acercan los acontecimientos a los sujetos receptores facilitando la experimentación a distancia de situaciones de inestabilidad social

Por último, los documentos institucionales que tratan sobre las formas de afrontar y comunicar sobre los riesgos, como es el caso del Marco de Sendai, atribuyen papeles muy vagos a la comunicación. Es necesario, entonces, tener muy en cuenta las diferentes instancias y el papel que cada una de ellas tiene en el proceso de comunicación de riesgos y catástrofes.

\section{Referências}

DELUMEAU, Jean. El miedo en Occidente. Madrid: Taurus, 2002.

GIL CALVO, Enrique. Riesgo, incertidumbre y medios de comunicación. In: MORENO CASTRO, Carolina. Comunicar los riesgos. Ciencia y tecnología en la sociedad de la información. Madrid: Organización de los Estados Iberoamericanos; Biblioteca Nueva, 2009. p. 185-197.

MARCO DE SENDAI PARA LA REDUCCIÓN DEL RIESGO DE DESASTRES 2015-2030. UNISDR: Ginebra, 2015.

PIÑUEL RAIGADA, José Luis; LOZANO ASCENCIO, Carlos. Ensayo general sobre la comunicación. Barcelona: Paidós, 2006. 
VICTOR, Cilene. Comunicação de riscos de desastres no contexto das mudanças climáticas: muito além do jornalismo. In: CONGRESSO BRASILEIRO DE CIÊNCIAS DA COMUNICAÇÃO, 37., 2015, São Paulo. Anais... São Paulo: INTERCOM, 2015.

\title{
Resumo
}

Este texto oferece uma abordagem teórica para apoiar um modelo de Comunicação Social de riscos e incertezas que, por um lado, estruture os vários passos necessários para identificar e definir a comunicação de riscos e de incertezas e, por outro, examine o papel da comunicação em abordagens como a do Marco de Sendai para a Redução do Risco de Desastres. A cada época correspondem diferentes formas de perceber e prever a instabilidade social para denominá-la e construi-la de forma significativa como um espaço do entorno circundante que demanda a máxima atenção dos sujeitos. Atualmente, é comum que se fale dos riscos para caracterizar nossas sociedades, mas nesse tipo de generalização costuma-se referir a situações de instabilidade muito mais complexas do que parecem e que nem sempre foram capazes de serem controladas somente com a previsão. Os conhecimentos e tecnologias não têm reduzido os níveis de fragilidade social e, mais ainda, as apreciações que fazem os indivíduos sobre as situações instáveis costumam incrementar historicamente os níveis de vulnerabilidade real, sobretudo porque se deixam influenciar pelo ponto de vista dos principais mediadores sociais.

\section{Palavras-chave}

Comunicação. Riscos. Sociedade de incertezas. Marco de Sendai.

\begin{abstract}
This text offers a theoretical approach to support a model of Social Communication of Risks and Uncertainties that, on the one hand, structures the different stages necessary to delimit and define "the knowledge of communication of risks and uncertainties" and, on the other, contribute to the Development of the Sendai Framework for Disaster Risk Reduction. At each epoch there are various ways of perceiving and predicting social instability to name it and constructing it significantly as a space in the surrounding environment that demands attention from the individuals'. At the moment, it is usual to talk about the risks to characterize our societies, but this kind of generalizations it is usually referred to situations of instability that are much more complex than they seem and that have not always been defined nor have been controlled with sole foresight. Knowledge and technology have not reduced levels of social fragility and, moreover, the estimations that individuals make about unstable situations usually increase historically the levels of real
\end{abstract}


vulnerability, especially because they are influenced by the point of view of the main social mediators.

\section{Keywords}

Communication. Risks. Society of uncertainty. Mark of Sendai.

Recebido em 24/11/2016

Aceito em 20/12/2016 